\title{
A case of prosthetic valve endocarditis caused by Corynebacterium hofmanni and Candida albicans
}

\author{
J. M. H. Boyce \\ From the Department of Bacteriology, West Cardiff Area Laboratory, St. David's Hospital, Cardiff
}

The case is described of a 49-year-old woman who developed endocarditis in relation to the prosthetic valve two months after insertion of a Starr-Edwards mitral valve prosthesis. Initially Corynebacterium hofmanni was isolated from blood culture. Later, while the patient was receiving antibacterial chemotherapy, Candida albicans was also isolated and she was treated with 5-fluorocytosine. A cure was effected without the need for further valve replacement.

A number of cases of prosthetic heart valve candida endocarditis successfully treated with antifungal therapy together with replacement of the prosthetic valve have been reported (English and Ross, 1972; Warner et al., 1970). The following case is unusual in that it represents a cure of candida endocarditis without the need for prosthetic valve replacement, and also in that the fungal endocarditis occurred concurrently with a bacterial endocarditis undergoing antibiotic chemotherapy.

\section{Case report}

A woman aged 49 years underwent insertion of a StarrEdwards mitral valve prosthesis in September 1972 for mitral stenosis and regurgitation. Antibiotic cover for the operation was ampicillin and cloxacillin.

During operation, she suffered a 3-hour period of severe hypotension due to mechanical problems causing poor perfusion which could not be overcome, and a tear in the back of the left ventricle, a well-known complication of mitral valve replacement, which caused considerable bleeding after coming off bypass. Once bleeding had been controlled, the blood pressure recovered and the circulation remained excellent in the postoperative period. However, cerebral deterioration developed 48 hours after operation and was probably due to a combination of the hypotension in the operative period and some postoperative hypoxia due to an intrapulmonary shunt associated with radiological changes of pulmonary oedema. She became aphasic and very drowsy and required mechanically-assisted respiration for 20 days from the date of operation during which time tetracycline and cloxacillin at different times in short courses were given for chest infection. Four weeks later in October she developed a subarachnoid haemorrhage, at a time when anticoagulant control was very difficult, and a left hemiparesis developed over the next 24 hours.
This hemiparesis gradually improved from about mid November.

A low grade pyrexia of $37.5^{\circ}-38^{\circ} \mathrm{C}$ developed from 4 November and from 14 November Corynebacterium hofmanni was isolated on 3 different occasions from a total of 6 blood culture bottles (Fig.). While awaiting results of culture and sensitivity tests the patient was started on cephalexin on 14 November. The antibacterial treatment was changed to cephaloridine $6 \mathrm{~g} /$ day and gentamicin $0.8 \mathrm{mg} / \mathrm{kg} 8$-hourly on 20 November when sensitivity test results were available. At the same time her diuretic therapy was changed from frusemide to chlorothiazide with potassium supplements. She remained pyrexial, though the bactericidal activity of her serum against the diphtheroid suggested that adequate levels of antibiotics were obtained. However, by 9 December she was in severe left heart failure, with nausea, vomiting, shortness of breath, and potassium depletion. Antibiotics were changed to clindamycin 300 $\mathrm{mg}$ 6-hourly and fusidic acid $500 \mathrm{mg} 8$-hourly, which was followed by a lowering of temperature though her heart failure remained uncontrolled. She progressively deteriorated showing increasing cardiac failure with oliguria and 4 fingers' breadth tender hepatomegaly. Blood cultures remained negative until 23 December when Candida albicans was isolated from 2 out of 3 blood culture bottles taken on 2I December. At about this time C. albicans was also isolated in moderate growth from urine, high vaginal swab, and sputum. While awaiting candida sensitivity tests she was immediately started on 5 -fluorocytosine (5-FC) $100 \mathrm{mg} / \mathrm{kg}$ per day. On 26 December improvement was noticed, with urine output increasing, breathing much easier, and her jugular venous pressure returning to normal. Her liver became non-tender and palpable at only $I$ finger breadth below the right costal margin. She became apyrexial from 29 December and remained so thereafter. The strain of candida isolated from blood culture was shown to be fully sensitive to $5-\mathrm{FC}$ at a minimal inhibitory 


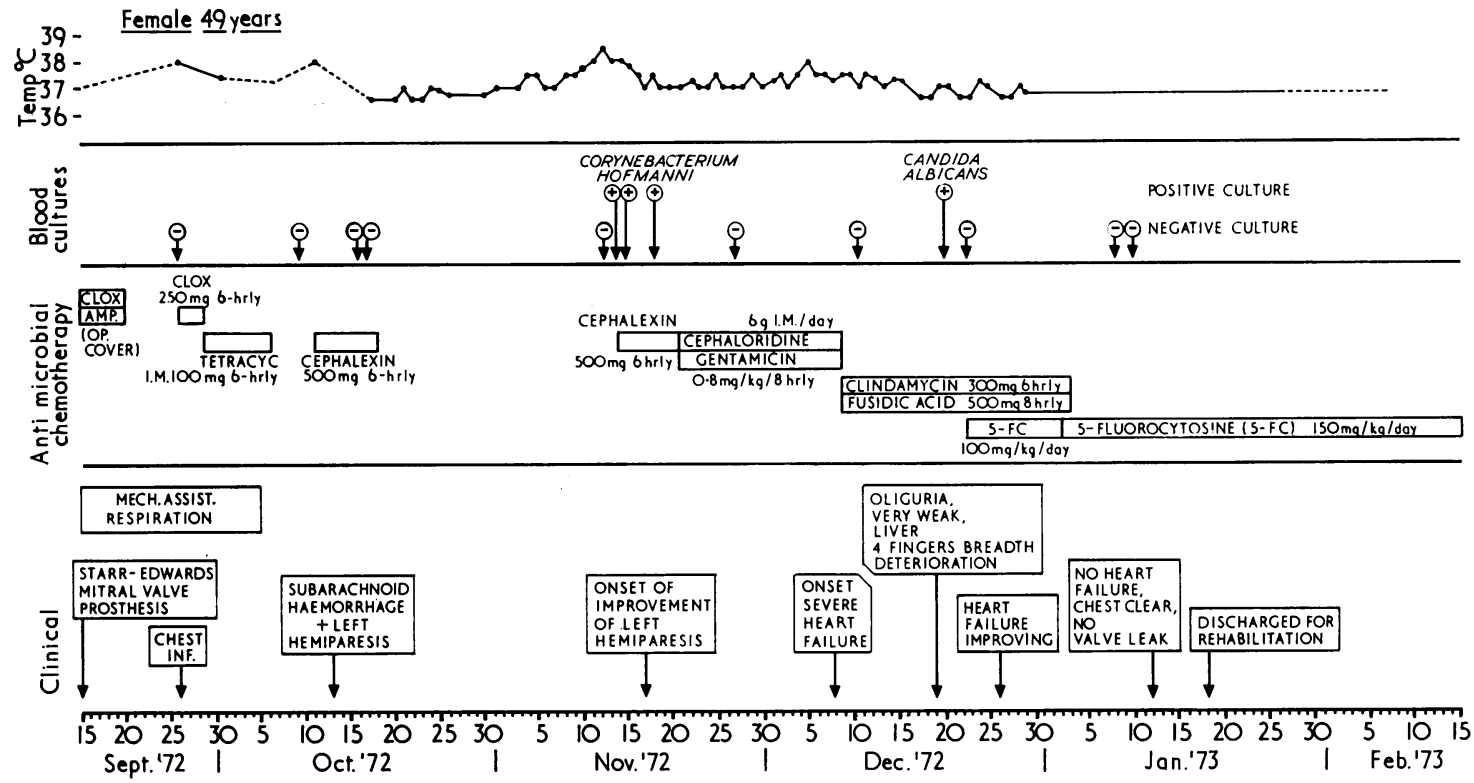

FIG. Summary of case history.

concentration of $0.1-0.2 \mu \mathrm{g} / \mathrm{ml}$, but dosage of this drug was increased to $150 \mathrm{mg} / \mathrm{kg}$ per day from 2 January 1973. Clindamycin and fusidic acid were stopped on 4 January - a total of 6 weeks of antibacterial therapy for the diphtheroid component of her endocarditis, and on 9 January, showing no evidence of heart failure or valve leak, she was discharged to another hospital for rehabilitation. 5-FC was continued until I6 February, maintaining satisfactory blood levels with no evidence of leucopenia, thrombocytopenia, or other side effects. Blood cultures taken during and after antifungal therapy showed no growth of bacteria or fungi. In May 1975 her cardiac condition remained good and there was no evidence of recurrence of her infective endocarditis.

\section{Comment}

The above case is instructive in that it demonstrates how a second infecting organism was responsible for the failure of response to treatment when all laboratory bacteriological tests indicated that the causal organism as primarily isolated should have been eradicated. Though it was difficult to decide which of the two organisms was primarily responsible for the infection, there was some improvement after treatment of the diphtheroid. The source of the candida could have been from urinary, genital, or respiratory tract as these areas revealed a moderate growth of Candida albicans shortly before blood cultures became positive. The successful outcome of this case may have been because of the relatively short time (as judged by repeatedly negative blood cultures until 21 December) that candida infection of the prosthetic valve was present.

The involvement of the prosthesis by candida can only be presumptive though there was no explanation for her continuing illness and severe progressive congestive cardiac failure other than the probability of fungal vegetation occlusion of the mitral valve, and her condition improved dramatically within a very short time of starting treatment with $5-\mathrm{FC}$.

The case is also interesting as it is an example of a cure, as judged by follow-up at 15 months after cessation of antifungal therapy, of a candida endocarditis upon a prosthetic heart valve without the need for replacement of the prosthesis. There have been very few reports of a cure by antifungal therapy alone of candida infection of prosthetic heart valves (Record et al., I97I ; S. B. Shohet, personal communication). Replacement of the prosthesis was not contemplated in our patient on account of the cerebral damage related to the first valve replacement.

Because 5-fluorocytosine is excreted entirely through the kidneys, renal function was carefully monitored during therapy as were 5-FC serum levels so that dosage adjustments could be made if impaired function developed. However, renal function in this patient remained satisfactory throughout. 
I am grateful to Mr. H. R. S. Harley, Consultant Cardiothoracic surgeon, for his permission to publish this case, and to Dr. R. J. Holt of Carshalton who kindly carried out sensitivity tests of the Candida albicans stain.

\section{References}

English, T. A. H., and Ross, J. K. (1972). Surgical aspects of bacterial endocarditis. British Medical fournal, 4, 598.

Record, C. O., Skinner, J. M., Sleight, P., and Speller, D. C. E. (1971). Candida endocarditis treated with 5fluorocytosine. British Medical fournal, 1, 262.
Warner, J. F., Duma, R. J., McGehee, R. F., Shadomy, S., and Utz, J. P. (1970). 5-fluorocytosine in human candidiasis. In Antimicrobial Agents and Chemotherapy, p. 473. Ed. by G. L. Hobby. American Society for Microbiology, Bethesda, Maryland.

Requests for reprints to Dr. J. M. H. Boyce, Department of Bacteriology, West Cardiff Area Laboratory, St. David's Hospital, Cowbridge Road East, Cardiff CFr 9TZ. 